\title{
La gestión de la convivencia escolar en la educación básica en México: desde la perspectiva del marketing educativo
}

\author{
The management of school coexistence in basic education in Mexico: \\ From the perspective of educational marketing
}

\author{
Emigdio Larios-Gómez ${ }^{1 \mathrm{a}}$ \\ Benemerita Universidad Autónoma de Puebla, México ${ }^{1}$ \\ D Orcid: https://orcid.org/0000-0002-3514-1319 ${ }^{1}$
}

Recibido: 08 de setiembre de 2020

Aceptado: 03 de diciembre de 2020

\begin{abstract}
Resumen
El objetivo del trabajo fue evaluar el clima escolar percibido (escolar y de aula) en la escuela secundaria de Amozoc de Mota (Puebla, México) desde una perspectiva del marketing educativo. Se desarrolló una investigación de tipo concluyente-descriptiva y transversal, encuestando de manera presencial a 215 docentes con un instrumento adaptando lo constructos sobre el Estudio de conductas, clima y convivencia escolar. Asimismo, se trató del análisis del ambiente escolar encuestando a 150 estudiantes. En los resultados analizados se pudo observar que el profesor de secundaria no percibe apoyo ni se siente valorado por parte de sus pares y directivos, lo que genera un clima laboral complejo e influye en una gestión difícil para la convivencia escolar. Se concluye que el profesor es un agente de andamiaje en el clima escolar, permite establecer en el Aula un espacio de instrucción y de construcción del aprendizaje con vínculos positivos, clima cordial y de seguridad con reglas y límites. Asimismo, promueve en los alumnos el desarrollo-aprendizaje personal, social académico y cognitivo para la vida. Además, el rol del docente dentro del aula es de primordial importancia en el clima de aula que perciban los alumnos. Los alumnos se sienten bien entre ellos, aceptados y respetados con base en la postura conciliadora, interventora y de autoridad del profesor. Y es donde el Marketing Educativo cumple su función y puede ayudar a mejorar la convivencia y promover las relaciones basadas en el respeto y la aceptación. Lo que desarrollará en los alumnos, docentes, directivos y padres de familia actitudes asertivas, críticas, con valores y conciencia analítica.
\end{abstract}

Palabras Clave: convivencia escolar; gestión escolar; clima escolar; clima de aula; marketing educativo 


\begin{abstract}
The objective of the work was to evaluate the Perceived School Climate (school and classroom) in the Amozoc de Mota secondary school (Puebla, Mexico) from an educational marketing perspective. It was developed a conclusive-descriptive and transversal research, surveying 215 teachers with an instrument adapting the constructs on the Study of behaviors, climate and school coexistence. Likewise, the analysis of the school environment was carried out by surveying 150 students. In the results analyzed, it was observed that the secondary school teacher does not perceive support or feel valued by his peers and directors, which generates a complex work environment and influences a difficult management for school coexistence. It was concluded that the teacher is an agent of scaffolding in the school climate, allowing the establishment of an instructional space in the classroom and the construction of learning with positive ties, a cordial and safe climate with rules and limits. Likewise, it promotes in the students the personal, social, academic and cognitive development-learning for life. In addition, the role of the teacher within the classroom is of paramount importance in the classroom climate perceived by the students. Students feel good about each other, accepted and respected based on the teacher's conciliatory, intervening and authoritative stance. This is where Educational Marketing comes in and can help improve coexistence and promote relationships based on respect and acceptance. This will develop in students, teachers, managers and parents assertive and critical attitudes, with values and analytical awareness.
\end{abstract}

Keywords: school life; School Management; school climate; classroom climate; educational marketing

\title{
Introducción
}

La demanda de profesionales sensibles y comprometidos, que tengan una formación que ayude a adaptarse a los nuevos requerimientos socio-laborales del entorno (Montes y Suárez, 2016) y a la convivencia social (Konishi et al., 2017), exige a las instituciones de Educación Básica (IEB) como a las Instituciones de Educación Superior (IES), que a partir del desarrollo de competencias (Braghirolli, Ribeiro, Weise y Pizzolato, 2016) -genéricas, técnicas, profesionales y blandas- desarrollen las habilidades innovadoras como pensamiento crítico, creatividad, colaboración y comunicación (Qian y Clark, 2016) en los estudiantes desde sus inicios académicos con el objetivo de resolver problemas y tomar decisiones en los diferentes campos de profesionales, empresariales, políticos y religiosos. Es así, que el desarrollo de competencias en las aulas se ha visto limitado en la clase debido a que se emplean técnicas y estrategias tradicionales que limitan el aprendizaje de la decisiones y cierra la visión ante los cambios del entorno (Brennan y Vos, 2013), ya que estas técnicas sólo involucran el desarrollo del área cognitiva de los estudiantes (Dávila y Velásquez, 2007) y no el desarrollo de las competencias sociales para una convivencia en un ambiente sano, seguro y libre de violencia (Larios-Gómez, Larios y Peña, 2018). 
El Plan Nacional de Desarrollo 2013-2018 (PND), en México establece el promover ambientes seguros para el estudio, en todas las escuelas del país. Así como fomentar la sana convivencia en un ambiente sano previniendo las situaciones de acoso escolar (PND, 2013), como principales líneas de acción en el rubro de educación, respecto al Clima Escolar. Por lo que el Gobierno Federal en México, por medio de la Secretaría de Educación Pública (SEP), estableció una Política Nacional para contar con escuelas libre de acoso escolar y una buena convivencia para la mejora educativa. Es así, que a partir del ciclo escolar 2016-2017, la Subsecretaría de Educación Básica (SEB), implementó el Programa Nacional de Convivencia Escolar (PNCE) con el propósito de garantizar las condiciones óptimas para una convivencia en la escuela y para un clima favorable en beneficio del aprendizaje. Teniendo como objetivo: "Favorecer el establecimiento de ambientes de convivencia escolar armónica, pacífica e incluyente, que coadyuven a prevenir situaciones de acoso escolar en escuelas públicas de educación básica, propiciando condiciones para mejorar el aprovechamiento escolar" (PNCE, 2017, p. 5) y sus objetivos específicos son:

1) Promover el desarrollo de ambientes adecuados para optimizar los procesos aprendizaje de las y los alumnos, 2) Proporcionar herramientas a los docentes para fomentar el desarrollo de las habilidades sociales y emocionales de las y los alumnos de educación básica, 3) Fortalecer la autoestima, la autorregulación de las emociones, el aprecio por la diversidad, el respeto a las reglas, la toma de acuerdos, la resolución pacífica de conflictos y el fortalecimiento de los valores familiares y 4) Promover la participación de las familias en la creación de ambientes escolares que contribuyan a la convivencia pacífica y al desarrollo integral de los alumnos (p. 6).

Las estrategias de acción que se han propuesto para la convivencia escolar en el PNCE, se tienen cinco directrices (SEB, 2016): 1) Las habilidades sociales y emocionales y su desarrollo., 2) Las emociones con su expresión y manejo (Trabajo integral y de desarrollo con docentes), 3) El manejo de conflictos a través del diálogo y acuerdos (Vinculación Interinstitucional), 4) El aprecio por la diversidad y 5) El fortalecimiento de los valores familiares (Trabajo integral con la familia). Y lleva ya cuatro años de existencia, y desde entonces implementa diagnósticos sobre el clima escolar, lo han favorecido el clima escolar y el aprendizaje de los estudiantes. El inicio fue con los ciclos escolares 2014-2016, con el Proyecto a favor de la Convivencia Escolar y su implementación en $3^{\circ}$ de primaria. Para el ciclo escolar 2016-2017 se implementó en el mismo nivel escolar, ahora para primaria de $1^{\circ} \mathrm{a} 6^{\circ}$ grado. En el ciclo escolar 2017-2018, la implementación abarco los niveles de preescolar, primaria y secundaria. Desde el ciclo escolar 2018-2019, la Convivencia Escolar favorece ambientes de aprendizaje seguros y libres de acoso y de violencia, coadyuva al desarrollo 
personal y social en el desarrollo emocional (desarrollo socioemocional), con autonomía curricular (Aguilera, 2015).

En Instituto Nacional para la Evaluación de la Educación (INEE) en México, ha estudiado la violencia en la convivencia escolar como un elemento de la calidad educativa desde dos perspectivas: 1) Desde el contexto para la enseñanza y el aprendizaje, como son las características de la escuela, las prácticas educativas, los recursos y lo procesos para la enseñanza y el aprendizaje y 2) Desde el resultado del aprendizaje. Es decir, el desarrollo de habilidades para aprender a convivir.

\section{Revisión de la literatura}

\section{Clima Escolar Percibido (escolar y de aula)}

Desde hace aproximadamente diez años, la investigación educativa en México contaba con pocas investigaciones de tipo cuantitativo sobre temas de calidad educativa, gestión educativa y más recientemente sobre la convivencia escolar. Para propósitos de la presente investigación, se abordará la dimensión sobre Clima Escolar exclusivamente y se dejarán las otras dimensiones para investigaciones futuras. Desde Edmonds (1979); Lee y Bryk (1989) y Lee et al. (1997), como pioneros, se ha mostrado que el Clima Escolar impacta en la calidad y la equidad educativa y construye las relaciones entre los agentes educativos (Loukas \& Murphy, 2007), y abarca aspectos sobre los procesos de comunicación y resolución de conflictos. Es decir, entre directivo-docente, lo que favorece la seguridad a los alumnos a través del respeto y la convivencia con la menor frecuencia de comportamientos violentos entre los pares (Greene, 2006; Stephenson y Smith, 2008; Johnson, 2009; Collie, Shapka y Perry, 2011; Good, McIntosch y Gietz, 2011). Y desde estas perspectivas, se puede observar a la convivencia escolar como un proceso interaccional personal y subjetivo (de alumnos, profesores y directivos) de una institución enmarcada por un conjunto de políticas y prácticas (involucradas en el proceso enseñanza-aprendizaje), que configuran y enmarcan este mismo proceso en sus dimensiones y escalas de los involucrados de la convivencia escolar. Y las Dimensiones del Clima escolar son: 1) Manejo de conflictos, 2) Participación de los estudiantes, 3) Trato inclusivo y 4) Disciplina escolar, que determinan los sujetos involucrados y su relación con otros sujetos, como se puede observar en la Tabla 1. 
Tabla 1

Dimensiones y Escalas de la Convivencia Escolar

\begin{tabular}{ll}
\hline Dimensiones & Escalas \\
\hline Clima escolar & Relación entre docentes y estudiantes \\
& Relación entre estudiantes \\
& Relación entre docentes \\
& Intervención docente para el dialogo y el entendimiento \\
Manejo de conflictos & mutuo \\
& Participación de los estudiantes en el aula \\
Participación de los & Participación de los estudiantes en la escuela \\
estudiantes & Trato inclusivo entre los estudiantes \\
Trato inclusivo & Prácticas disciplinarias que atentan contra los derechos de los \\
Disciplina escolar & estudiantes \\
\hline
\end{tabular}

Fuente: Aguilera (2015).

Asimismo, el clima escolar, se considera predictor en los resultados académicos de los estudiantes (Benbenishty et al., 2016), así como un ajuste en la vida escolar del alumno, desde un enfoque psicológico (Voight y Nation, 2016). Y pone de relieve la importancia de la calidad entre las interacciones (bienestar y su sentimiento de aceptación y valoración por los demás), de los estudiantes y de estos entre los docentes (Oliva et al., 2009). Espelage, Low y Jimerson (2014); Konishi et al. (2017); Low y Van Ryzin (2014) concluyen que el clima escolar se relaciona con el bienestar del alumno, con los resultados académicos y con las conductas y actitudes de acoso. Desde esta perspectiva, también es considerado como factor en la influencia en el logro académico de los alumnos en la escuela y, por tanto, en la calidad y equidad de los resultados educativos tanto en el aula, como en la escuela en su inmersión global (Blanco, 2009; Juárez y Fierro, 2013), con las relaciones interpersonales en el aula, disciplina escolar y resolución de conflictos. Y varios de estos aspectos están relacionados en el contexto actual con la convivencia escolar efectiva, que en décadas pasadas se asociaban al currículo oculto.

Finalmente, el clima escolar debe promover el desarrollo de los alumnos desde una perspectiva personal, social y académica. Debe facilitar el establecimiento de vínculos positivos entre los alumnos y los profesores. Así como también debe promover un clima afectuoso y de seguridad, con normas y límites claros, sin descuidar la proximidad afectiva en las relaciones y el sentimiento de autovaloración, pertenencia y orgullo (Oliva, 2015; Pertegal et al., 2015). Entonces, para construir un clima social-escolar afectivo, se requiere de convenciones sociales y hábitos de cortesía, la promoción de valores éticos y el cuidado del otro (en la convivencia) y las relaciones familiares (en la administración y sostenimiento de la escuela (Landeros y Chávez, 2015). 
Voight y Nation (2016) proponen una serie de dimensiones que integran el clima escolar en la escuela, que inciden las prácticas y los programas académicos, en pro del el rendimiento y el bienestar del alumno: relaciones interpersonales, disciplina, ambiente físico, seguridad, participación y respeto a la diversidad. Más reciente, Konishi, Miyazaki, Hymel y Waterhouse (2017), proponen dimensiones desde el enfoque académico, social, físico (seguridad) y afectivo (vinculación). Así también, en México para el INEE (2018; 2019), las dimensiones del Clima Escolar son la relación que se da entre profesores y los estudiantes, la relación que se da sólo entre los estudiantes y la relación que se da sólo entre profesores, ver Figura 1.

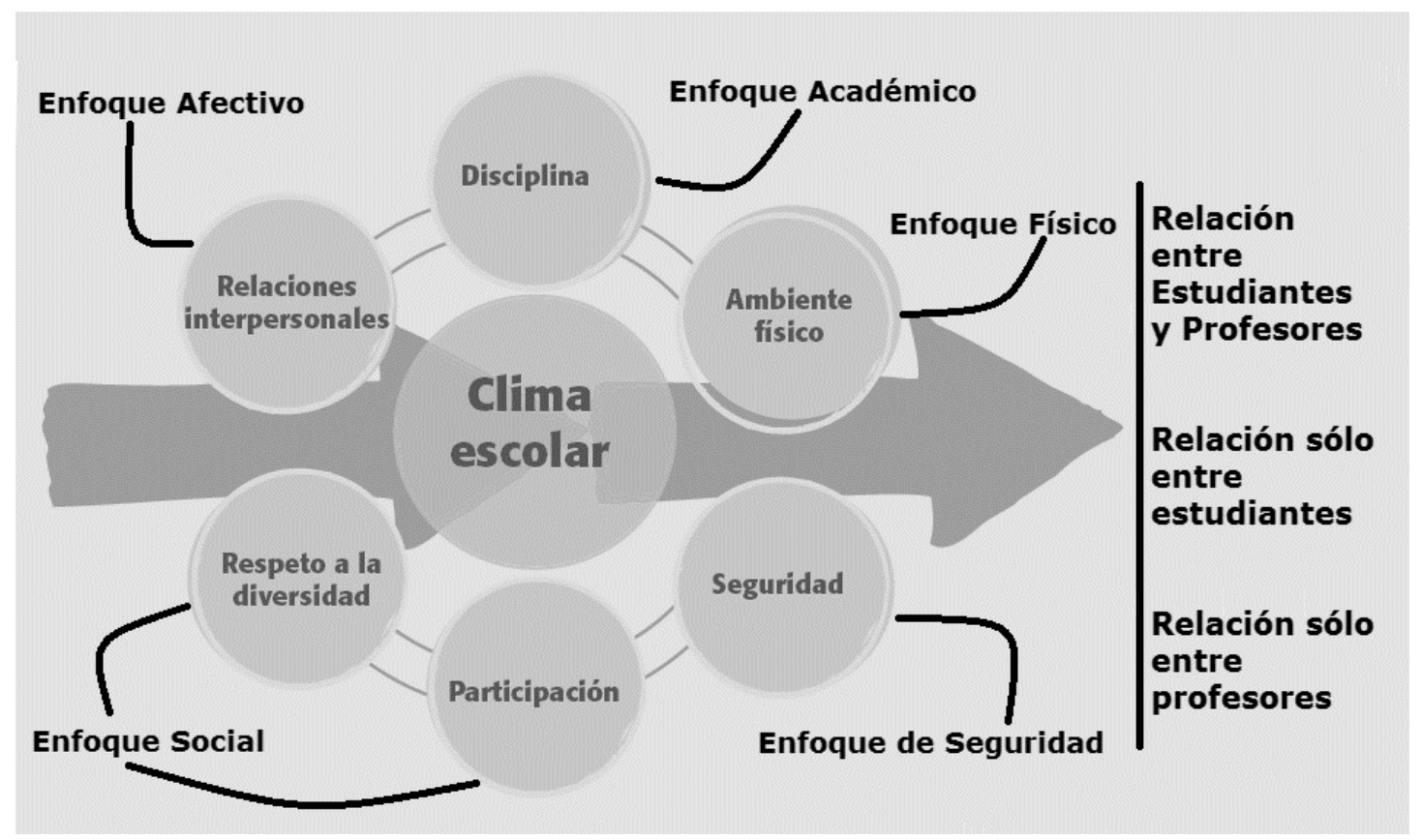

Figura. 1. Dimensiones del Clima Escolar (Adaptado de Voight y Nation 2016; Konishi, Miyazaki, Hymel y Waterhouse, 2017; INEE; 2018; 2019).

Blanco (2009) en sus estudios sobre el Clima Social Escolar, lo divide en dos subtemas para su mejor análisis: 1) en el mismo clima escolar y 2) en el clima de aula. Ambos como factor de gran importante para la calidad educativa y como sistemas de representaciones donde interactúan los actores escolares en el proceso enseñanza-aprendizaje (el proceso educativo, el trabajo grupal y las interacciones entre docente-alumno y directivo-docente). Describe como Clima Escolar las representaciones que se dan entre los significados compartidos por los maestros y el directivo y describe al clima de aula como las representaciones significativas que comparten los alumnos de un grupo y su maestro (2010). Y lo divide en dos subtemas para su mejor análisis: 1) en el mismo clima escolar y 2) en el clima de aula. Este último, tiene dos 
dimensiones desde la percepción de los profesores y desde la percepción de los alumnos, y se entiende como las relaciones entre los alumnos y su profesor ya sea el tutor del grupo o del profesor de cualquier asignatura dentro del Aula (salón o laboratorio), que afecta significativamente la autoconcepción de los alumnos y su nivel de aprendizaje (Link y Ratledge 1975; Alexander et al. 1987). Debido a que el Aula es un espacio de instrucción y de construcción del aprendizaje y de las relaciones sociales, ambos involucrados en el proceso educativo y sus resultados en el aprendizaje social, educativo, cognitivo y para la misma vida.

Si bien, la unidad de análisis en el clima escolar es la escuela (con todos sus agentes involucrados). El proceso educativo realmente se da dentro de aulas, mediante las representaciones e interacciones entre el (tutor) o los profesores (diferentes asignaturas) y sus alumnos, y aislados muchas de las veces de los grupos (aulas) restantes, ya sean del mismo grado o grados inferiores o superiores. Entonces, desde la perspectiva de Blanco (2009), de Voight y Nation (2016), de Konishi et al., (2017), del INEE (2018; 2019) y de Landeros y Chávez (2015), el aula también es una unidad de análisis -como la misma escuela en su totalidad y el grupo de padres de familia por aparte- en el clima social escolar. Las relaciones entre los alumnos y su profesor ya sea el tutor del grupo o del profesor de cualquier asignatura dentro del Aula (salón o laboratorio), afectan significativamente la autoconcepción de los alumnos y su nivel de aprendizaje (Link y Ratledge 1975; Alexander et al. 1987). Debido a que el Aula es un espacio de instrucción y de construcción del aprendizaje y de las relaciones sociales, ambos involucrados en el proceso educativo y sus resultados en el aprendizaje social, educativo, cognitivo y para la misma vida.

\section{El marketing educativo}

El marketing en colegios y universidades se ha aplicado en los últimos años veinte años (Wasner \& Bruner, 2000), debido a la competencia en las instituciones de educación -básica media y superior- lo que ha forzado a satisfacer al cliente [estudiantes reales y estudiantes potenciales, y padres de familia] (Kotler 2000; Schultz, 2002). A lo que se le ha llamado marketing o mercadotecnia educativa, teniendo origen en la mercadotecnia social (RussellBennett et al., 2016), aunque también tiene un origen desde su objetivo comercial: el de posicionar y vender los servicios educativos. Manes (2005), argumenta que es necesario que la Institución Educativa (IE), pública o privada, deba incorporar en su estructura al marketing como proceso y designar un responsable del área al servicio de la comunidad educativa. Por otro lado, Naranjo (2011) afirma que las estrategias del Marketing Educativo tienen como objetivo crear y facilitar a los clientes consumidores (estudiantes), puedan interactuar en 
situaciones de aprendizaje participativo dentro y fuera de contextos educativos, en su individualidad y diversidad. Finalmente, Larios-Gómez (2015, p. 10) define a la mercadotecnia educativa como

...la herramienta estratégica de la gestión, que ejecutan las instituciones de educación (de todos los niveles o grados escolares) y que permite satisfacer necesidades de desarrollo personal para la adquisición de nuevo conocimiento, a través de la comercialización (intercambio y venta) de un servicio [educativo], de forma directa o indirecta (presencial o virtual).

El campo de acción del marketing es holístico, que ha impactado no solo en las disciplinas que integran las ciencias administrativas y sociales, sino también en las disciplinas de las ciencias exactas. Asimismo, en la tecnología, en la religión, en los deportes y en la educación, entre otros campos de acción. El marketing se ha integrado a la educación desde la comercialización de los servicios educativos, en el estudio del clima laboral y hasta en el proceso enseñanza-aprendizaje. En este último, no es reciente, desde 1970 se ha visto reflejado en juegos, simuladores, apps, interacciones offline y outline con el consumidor de forma directa [BTL por sus siglas en inglés below the line] y en estudios del comportamiento del consumidor (Vos, 2015; McRaith y Goeldner, 1962; Forrester, 1959; March, 1962; Shycon y Maffei, 1960; Titus y Petroshius, 1993). Por lo que respecta al clima laboral de las organizaciones y en especial en las que se dedican a la educación, el marketing interno funciona como una herramienta de análisis y diagnóstico que enfatiza que los empleados son primer mercado de estudio para las IE (Wasner, Gordon y Bruner, 2000). Debe garantizar que los empleados (directivos, docentes, personal administrativo y de servicios de apoyo general) perciban que la dirección se preocupa por ellos y sus necesidades y una vez satisfechos se ofrezca un excelente servicio educativo a los clientes (Ewing y Caruana, 1999; Gronroos, 1990, 2000; Kotler, 2000; Caruana y Calleya, 1998).

Las IE públicas y privadas en México, estas últimas principalmente si ponen en práctica el marketing interno educativo, y se han percatado que la orientación del mercado se ha modificado en relación a los intercambios entre empleadores y empleados en el mercado interno (Lings y Greenley, 2005) e implica el desarrollo de una inteligencia emocional relacionada con los deseos y necesidades de los empleados (Johlke y Duhan, 2000), que se enfoca en los diferentes tipos de interacciones entre gerentes y el personal educativo, como a) la generación formal de información escrita a través de los medios oficiales de la IE, b) la generación formal de información cara a cara en la interacción interna y c) la generación informal de información cara a cara que se da fuera de los contextos laborales y en menor proporción dentro del mismo 
(Santos y Gonçalves, 2018). Entonces, el Marketing Educativo Interno ayuda a mejorar la convivencia y promueve las relaciones basadas en el respeto y la aceptación, que aunado con los planes y programas educativos, y las estrategias de convivencia escolar los alumnos desarrollan actitudes asertivas, críticas y conciencia analítica (Tuvilla, 2004; Pineda-Alfonso y García-Pérez, 2016; Celkan et al., 2015).

\section{Materiales y métodos}

El presente trabajo de investigación tuvo el objetivo de evaluar el clima escolar percibido (CEP) integrado por el clima escolar y el clima de aula, en las escuelas que integran la supervisión de la Zona Escolar 038 de nivel secundaria en Amozoc de Mota en Puebla México desde una perspectiva del marketing educativo interno. Se trata de un estudio de tipo concluyente-descriptivo y transversal (Kerlinger y Lee, 2002; Malhotra, Kim y Patil, 2006), a través de la aplicación de una encuesta presencial a docentes y a alumnos. Se tomaron en cuenta la totalidad de las escuelas secundarias que integran el municipio (13 escuelas, es decir fue un censo) y con base en el ciclo escolar 2017-2018 la planta docente fue de 215 profesores (76 son hombres y 139 son mujeres). Y la muestra de alumnos fue por conveniencia, de 150 alumnos, por lo que la investigación es no probabilística.

Se aplicaron dos instrumentos tomados y adaptados del Estudio de conductas, clima escolar y convivencia en los centros escolares de Cantabria (Lázaro-Visa \& Fernández-Fuertes, 2018). La operacionalización del constructo en sus variables, se puede observar en la Tabla 2, para el instrumento A, aplicado a profesores integrado por 16 ítems en total, para medir el Clima Escolar Percibido (CEP) se emplean 8 ítems, en las relaciones entre profesores y sus directivos, se emplean 2 ítems, en las relaciones de la convivencia y el clima escolar y se emplean 6 ítems para medir la valoración del profesor en sus relaciones con los demás agentes en el clima escolar (alumnos, autoridades, directivos, compañeros de trabajo. Y el instrumento B aplicado a los alumnos integrado por 32 ítems, para medir el Clima de Aula Percibido (CAP) se emplean 14 ítems; para medir el autoconcepto (AC) se emplean 5 ítems y para para medir las normas de convivencia (NC) se emplean 13 ítems (desde la perspectiva del alumno). 
Tabla 2

Operacionalización de Variables: Clima Social Escolar

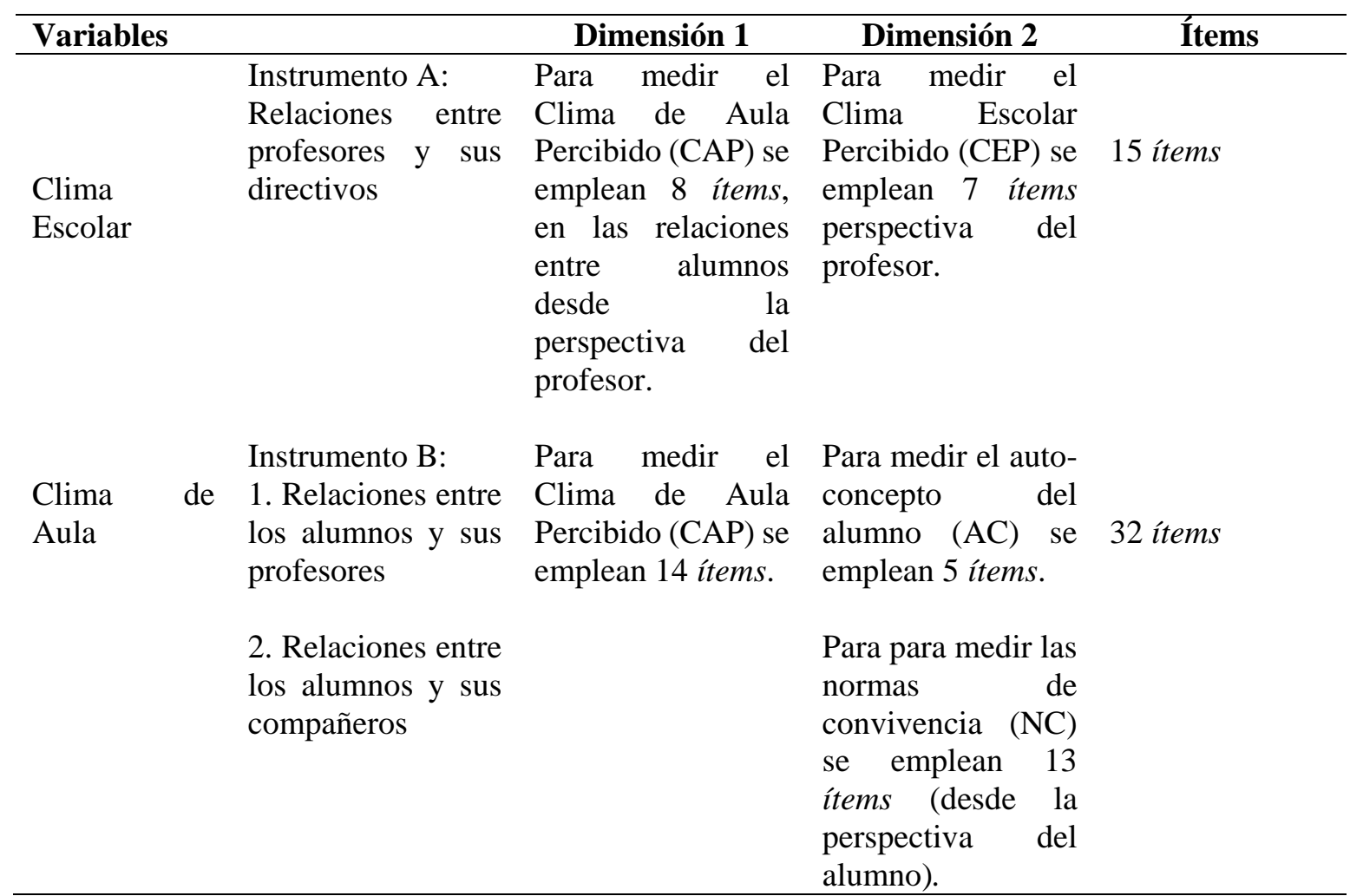

Fuente: Elaboración propia con información de (Lázaro-Visa \& Fernández-Fuertes, 2018).

\section{Resultados}

Se realizó la validación del instrumento con expertos y se modificó el ítem (CP_C4), que se repetía, ajustándolo respecto a la valoración de la labor docente del profesor de secundaria (padres de familia). Se calculó la consistencia interna basado en el Alfa de Cronbach, que permitió estimar la fiabilidad de los instrumentos, la cual fue de 0.965 para el instrumento A (profesores) y de 0.873 para el instrumento B (alumnos), considerado excelente y adecuado respectivamente (Nunnally, 1978).

\section{Análisis de Correlaciones (KMO y Test De Bartlett)}

Mateos y Martín (2002) describen que el KMO sirve para comparar las magnitudes de los coeficientes de correlación general o simple con respecto a las magnitudes de los coeficientes de correlación parcial. Al calcular el Índice Kaiser-Meyer-Olkin, se avaló la adecuación de la muestra y que tan apropiado fue aplicar el Análisis Factorial. Los valores entre 0.5 y 1 indican que es apropiado aplicarlo. Por lo que se ocupó el Test de Esfericidad de Bartlett para probar la Hipótesis Nula que afirma que las variables no están correlacionadas en la 
población. Es decir, si la matriz de correlaciones es una matriz de identidad, cuya fiabilidad sea menor a 0.05 (Montoya, 2007), como se muestra en la Tabla 3.

Tabla 3

Prueba de KMO y Bartlett

\begin{tabular}{cr}
\hline Medida Kaiser-Meyer-Olkin de adecuación de muestreo & .923 \\
Prueba de esfericidad de Bartlett Aprox. Chi-cuadrado & 4899.587 \\
Gl & 1256 \\
Sig. & .000 \\
\hline
\end{tabular}

Fuente: Elaboración propia con SPSS 25.

\section{Análisis del clima escolar desde la perspectiva de los profesores (Instrumento A)}

Como se puede observar, el nivel de correlación entre las variables y competitividad es aceptable conforme a los parámetros que fueron establecidos. De igual manera el nivel de significancia es óptimo porque presentó una significancia muy inferior al límite 0.05 , pues fue de 0.000 , lo cual nos indica que la matriz de datos es válida para continuar con el proceso de análisis factorial. También se muestra que el cuestionario presenta un amplio margen de los grados de libertad (1256) porque tiene más posibilidades de respuestas y por último la Chicuadrada nos dice que 4899.587 son los ítems útiles y son aprovechados un $92 \%$ de la varianza de los datos originales.

En la Tabla 4 se describe la medición del Clima de Aula Percibido (CAP) en el análisis de la correlación, en las tres correlaciones, existe una relación lineal significativa positiva alta (Johnson, 2003), entre el ítem CP_B2 y CP_A1 (con un coeficiente de Pearson $\mathrm{r}=.844^{* *}$ y significativa al $\mathrm{p}=0.000)$. 
Tabla 4

Correlación de los ítems: Docentes

\begin{tabular}{|c|c|c|c|c|c|c|}
\hline \multicolumn{7}{|c|}{ Correlaciones } \\
\hline \multirow{9}{*}{$\begin{array}{l}\text { Clima de } \\
\text { Aula } \\
\text { Percibido } \\
\text { (CAP) }\end{array}$} & \multirow{4}{*}{ CP_B2 } & & CP_B2 & CP_A1 & CP_A4 & CP_A7 \\
\hline & & Correlación de Pearson & 1 & $.844 * *$ & $.815^{* *}$ & $.869 * *$ \\
\hline & & Sig. (bilateral) & & .000 & .000 & .000 \\
\hline & & $\mathrm{N}$ & 215 & 215 & 215 & 215 \\
\hline & \multirow{2}{*}{\multicolumn{6}{|c|}{$\begin{array}{l}\text { **. La correlación es significativa al nivel 0,01 (bilateral). } \\
\text { CP B2 El clima de participación en sus clases es adecuado }\end{array}$}} \\
\hline & & & & & & \\
\hline & \multicolumn{6}{|c|}{ CP_A1 En clase predomina la competencia sobre la cooperación. } \\
\hline & \multicolumn{6}{|c|}{$\begin{array}{l}\text { CP_A4 Los niños/as agresivos y dominantes no les gusta respetar las normas de } \\
\text { convivencia. }\end{array}$} \\
\hline & CP_A7 & las relaciones entre los al & nos son be & & & \\
\hline
\end{tabular}

Clima de

Aula

Percibido CP_B1

(CAP)
Correlación de Pearson
Sig. (bilateral)
$\mathrm{N}$

$$
\begin{array}{rrr}
\text { CP_B1 } & \text { CP_A3 } & \text { CP_A6 } \\
1 & .796^{* *} & .800^{* *} \\
& .000 & .000 \\
215 & 215 & 215
\end{array}
$$

**. La correlación es significativa al nivel 0,01 (bilateral).

CP_B1 Valoración del clima de convivencia en sus clases

CP_A3 En el aula hay niños/as que muestran comportamientos intimidatorios sobre los demás.

CP_A6 En general, mi relación con los alumnos es satisfactoria.

Clima

Escolar

Percibido

(CEP).

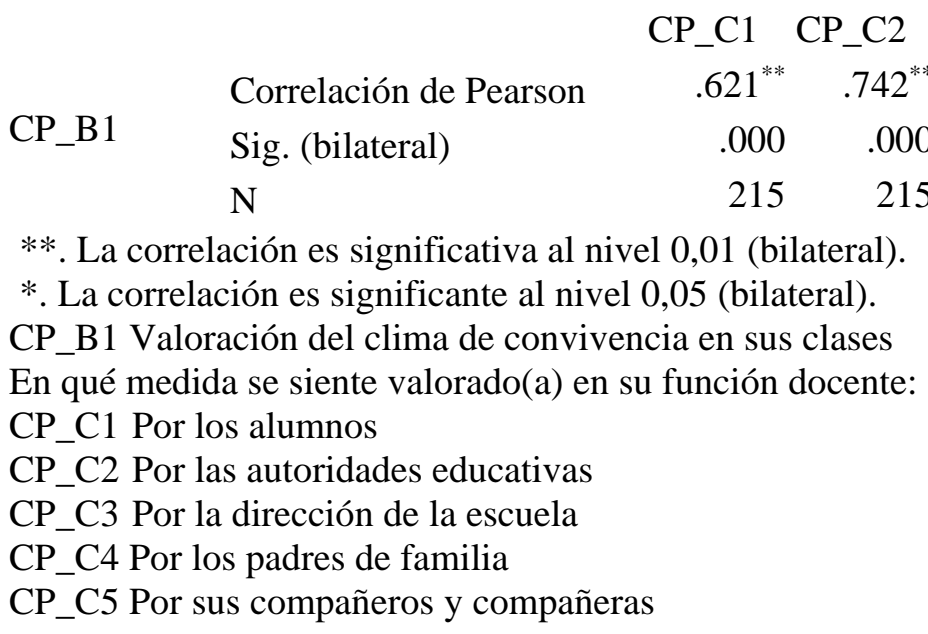

Clima

Escolar

Percibido

CP_B1

Correlación de Pearson

CP_B1 CP_C6

Sig. (bilateral)

$1.680^{* * *}$

(CEP).

**. La correlación es significativa al nivel 0,01 (bilateral).

*. La correlación es significante al nivel 0,05 (bilateral).

CP_B1 Valoración del clima de convivencia en sus clases

CP_C6 Grado de satisfacción que experimenta en el ejercicio de su labor profesional.

Fuente: Elaboración propia con SPSS 25. 
Entre los ítems CP_B2 y CP_A4 (con un coeficiente de Pearson $\mathrm{r}=.815^{* *}$ y significativa al $\mathrm{p}=0.000$ ) y finalmente entre los items CP_B2 y CP_A7 (con un coeficiente de Pearson $\mathrm{r}=.869^{*} *$ y significativa al $\mathrm{p}=0.000$ ). Es decir, desde la perspectiva del profesor de secundaria percibe que:

- Con base en los comportamientos de los alumnos de secundaria en la clase, existe un clima escolar de participación adecuado (en una escala del 1 al 5, la escala obtenida de 4 significa de acuerdo), es decir el $83.3 \%$ de los profesores lo percibe.

- Los datos están conservadoramente dispersos y concentrados cercanos al promedio respecto al .622, (Rango de 3= mínimo 2 y Máximo 5).

- Respecto al clima de participación en clases predomina la competencia sobre la cooperación entre los alumnos, con una media de 3.73 (ítem CP_A1), con el 76.2\% de la percepción de los profesores. Los datos están conservadoramente dispersos y concentrados cercanos al promedio respecto al .857, (Rango de 3= mínimo 2 y Máximo $5)$.

En el análisis del CAP, se detectó que el principal problema que presenta el clima escolar es que los niños/as agresivos y dominantes no les gusta respetar las normas de convivencia (ítem CP_A4), observación manifestada por el 64.3\% de los profesores (contra el 28.6 que no está de acuerdo) y con una media de 3.43, y presencia de datos altamente dispersos y concentrados cercanos al promedio respecto al 1.151, (Rango de 4= mínimo 1 y Máximo 5). Sin embargo, en general, desde la percepción de los profesores de secundaria, el $90.5 \%$ percibe que las relaciones entre los alumnos son buenas (ítem CP_A7), con una media 4.00 y los datos están conservadoramente dispersos y concentrados cercanos al promedio respecto al .541, (Rango de 3= mínimo 2 y Máximo 5).

Asimismo, en la Tabla 4 se describe la medición del Clima de Aula Percibido (CAP), en las relaciones entre alumnos desde la perspectiva del profesor. En el análisis de correlaciones 2, existe una relación lineal significativa positiva alta (Johnson, 2003), entre el ítem CP_B1 con los items CP_A3 (con un coeficiente de Pearson $\mathrm{r}=.796^{* *}$ y significativa al $\mathrm{p}=0.000$ ) y con CP_A6 (con un coeficiente de Pearson $\mathrm{r}=.800 * *$ y significativa al $\mathrm{p}=0.000$ ). Se puede interpretar que el clima de convivencia (CP_B1) de los alumnos en sus clases $(\mathrm{x}=4.3 \mathrm{y} \sigma=.570)$ y que se perciba en el aula que hay niños/as que muestran comportamientos intimidatorios sobre los demás $(\mathrm{x}=2.9$ y $\sigma=1.19)$. Es decir: 
- Del 95.2\% de los profesores que perciben una convivencia adecuada entre sus alumnos, el $47.7 \%$ percibe comportamientos intimidatorios. Nos que hace reflexionar sobre la existencia de casos de acoso escolar en la zona escolar 038 en Amozoc de Mota y su imperante intervención por parte de las autoridades y profesores.

- La medición del Clima Escolar Percibido (CEP), en las relaciones entre los profesores y los demás agentes de convivencia en la escuela secundaria (alumnos, directivos y padres de familia), se tiene que en el análisis de la medida de cómo se siente valorado(a) el profesor en su función docente.

La correlación 3a en la combinación ítem CP_B1 Valor del clima de convivencia y los ítems de la sección agentes del clima escolar (CP_C1, CP_C2, CP_C3, CP_C4 y CP_C5). Se sostiene que existe una relación lineal significativa positiva moderada (Johnson, 2003), en esta correlación: 1) CP_B1 y CP_C1 con un coeficiente de Pearson $\mathrm{r}=.621 * *$ y significativa al $\mathrm{p}=$ $0.000,2)$ CP_B1 y CP_C2 con un coeficiente de Pearson $r=.742 * *$ y significativa al $\mathrm{p}=0.000$, 3) $\mathrm{CP} \_\mathrm{B} 1$ y CP_C3 con un coeficiente de Pearson $\mathrm{r}=.735^{* *}$ y significativa al $\left.\mathrm{p}=0.000,4\right)$ CP_B1 y CP_C4 con un coeficiente de Pearson $\mathrm{r}=.498^{* *}$ y significativa al p=0.001 y 5) CP_B1 y CP_C5 con un coeficiente de Pearson $\mathrm{r}=.511 * *$ y significativa al $\mathrm{p}=0.001$.

Desde la perspectiva del profesor de secundaria en Amozoc de Mota (Puebla), del 95.2\% docentes que valoran el clima de convivencia escolar como adecuado en su escuela $(x=4.33$ y $\sigma=.570$ ) [De una escala del 1 al 5 . Donde 1 es muy en desacuerdo y 5 muy de acuerdo], es decir se sienten bien en su ambiente de trabajo, el 61.9\% perciben ser valorados como mucho [De una escala del 1 al 10. Donde 1 corresponde a nada y 10 corresponde a mucho] por los alumnos, en una escala de 8 a 10 (CP_B1 y CP_C1 con un coeficiente de Pearson r=.621** y significativa al $\mathrm{p}=0.000$ ). Parecido pasa o siente con sus compañeros (as), donde percibe que el $88 \%$ de sus compañeros de trabajo valoran como mucho su valor docente. Con una media $x=8.5$ y una desviación estándar $\sigma=1.08$, con presencia de datos altamente dispersos y concentrados cercanos al promedio (CP_B1 y CP_C5 con un coeficiente de Pearson $\mathrm{r}=.511 * *$ y significativa al $\mathrm{p}=0.001)$.

Para el caso de las autoridades educativas (supervisión) y autoridades de la escuela (director). Desde la perspectiva del profesor de secundaria en Amozoc de Mota (Puebla), del 95.2\% docentes que se sienten bien en su ambiente de trabajo, el 78.6\% perciben ser valorados como mucho por las autoridades educativas (supervisión), en una escala de 8 a 10 (CP_B1 y CP_C2 con un coeficiente de Pearson $\mathrm{r}=.742 * *$ y significativa al $\mathrm{p}=0.000$ ). Con una media $\mathrm{x}=$ 8.07 y una desviación estándar $\sigma=2.19$, con presencia de datos altamente dispersos y 
concentrados cercanos al promedio). Contrario al $11.1 \%$ valorado de regular (escala 7) y el $4.8 \%$ como neutro (escala 5). Respecto a las autoridades de la escuela (director), sucede que el 95.2\% perciben ser valorados como mucho por su director, en una escala de 8 a 10 (CP_B1 y CP_C3 con un coeficiente de Pearson $\mathrm{r}=.735^{* *}$ y significativa al $\mathrm{p}=0.000$ ). Contrariamente, el $2.4 \%$ se siente valorado como regular y en el mismo porcentaje, el $2.4 \%$ nada. Con una media $\mathrm{x}=8.9$ y una desviación estándar $\sigma=1.62$ con presencia de datos altamente dispersos y concentrados cercanos al promedio). Cabe señalar, que respecto a los padres de familia (desde la perspectiva de los que se involucran con la educación de su hijo), sucede que el profesor siente que solo en un $88.1 \%$ es valorado como mucho por los tutores o padres de familia de sus alumnos, en una escala de 8 a 10 . Contrario al $11.1 \%$ valorado de regular a neutro, en una escala de 5 a 7. La relación es entre el ítem CP_B1 y CP_C4 con un coeficiente de Pearson $\mathrm{r}=.498^{* *}$ y significativa al $\mathrm{p}=0.001$ ). Con una media $\mathrm{x}=8.5$ y una desviación estándar $\sigma=1.08$ con presencia de datos altamente dispersos y concentrados cercanos al promedio).

Finalmente, los datos correspondientes al Clima Escolar Percibido (CEP), la correlación 3b en la combinación ítem CP_B1 Valor del clima de convivencia escolar y 6) CP_C6 Grado de satisfacción en el ejercicio laboral. Existe una relación lineal significativa positiva moderada (Johnson, 2003) entre el ítem CP_B1 y CP_C6, con un coeficiente de Pearson r=.680** y significativa al $\mathrm{p}=0.000$, desde la perspectiva del profesor de secundaria en Amozoc de Mota (Puebla). Del 95.2\% docentes que se sienten bien en su ambiente de trabajo, el 100\% se siente satisfecho con su labor profesional como profesor, con un grado de satisfacción como mucho, en una escala de 8 a 10 (sólo la escala de 10 obtuvo el 61.9\%, la escala de 9 el $28.6 \%$ y la escala de 8 el 9.5\%). Con una media $\mathrm{x}=9.5$ y una desviación estándar $\sigma=.67$, con presencia de datos moderadamente dispersos y concentrados cercanos al promedio (Tabla 4).

\section{Análisis del clima escolar desde la perspectiva del Alumno (Instrumento B)}

En la Tabla 5, se describe la medición del Clima de Aula (CA), en las relaciones entre los profesores y sus alumnos, como agentes de convivencia en la escuela secundaria, se tiene que en el análisis del Clima de Aula desde la perspectiva del alumno. La correlación múltiple 1 en la combinación ítem CA_D1 En clase tengo (amigos) y los ítems de la dimensión Clima de Aula Percibido por el Alumno (CAPA): CA_E1, CA_E2, CA_E3, CA_E4 y CA1_F1. Se sostiene que existe una relación lineal significativa positiva alta (Johnson, 2003), en esta correlación: 1) CA_D1 y CA_E1 con un coeficiente de Pearson $\mathrm{r}=.835 * *$ y significativa al $\mathrm{p}=$ $0.000,2)$ CA_D1 y CA_E2 con un coeficiente de Pearson $\mathrm{r}=.834 * *$ y significativa al $\mathrm{p}=0.000$, 
3) CA_D1 y CA_E3 con un coeficiente de Pearson $r=.819^{* *}$ y significativa al $\mathrm{p}=0.000,4$ )

CA_D1 y CA_E4 con un coeficiente de Pearson $r=.830 * *$ y significativa al $\mathrm{p}=0.000$ y 5)

CA_D1 y CA1_F1 con un coeficiente de Pearson $\mathrm{r}=.740 * *$ y significativa al $\mathrm{p}=0.001$.

Tabla 5

Correlación: Alumno (CAPA).

\begin{tabular}{|c|c|c|c|c|c|c|c|}
\hline & & CA_D1 & CA_E1 & CA_E2 & CA_E3 & CA_E4 & CA1_F1 \\
\hline \multirow{5}{*}{ CA_D1 } & Correlación de & 1 & $.835^{* *}$ & $.834^{* * *}$ & $.819^{* *}$ & $.830^{* * *}$ & $.740^{* *}$ \\
\hline & Pearson & & & & & & \\
\hline & Sig. (bilateral) & & .000 & .000 & .000 & .001 & .001 \\
\hline & $\mathrm{N}$ & 115 & 115 & 115 & 115 & 115 & 115 \\
\hline & $\begin{array}{l}\text { **. La correlac } \\
* \text {. La correlació } \\
\text { CA_D1 En clas } \\
\text { CA_E1 ¿Te deja } \\
\text { CA_E2 En la cl } \\
\text { CA_E3 Los pro } \\
\text { CA E4 Con los }\end{array}$ & $\begin{array}{l}\text { significa } \\
\text { ignifican } \\
\text { o (amigo } \\
\text { compañe } \\
\text { profesor } \\
\text { s nos ens } \\
\text { añeros de }\end{array}$ & $\begin{array}{l}\text { a al nivel } \\
\text { al nivel } 0 \\
\text { s jugar co } \\
\text { s anima e } \\
\text { lan a lleva } \\
\text { lase me } 1\end{array}$ & $\begin{array}{l}0,01 \text { (bil } \\
05 \text { (bilat } \\
\text { n ellos? } \\
\text { n los trab } \\
\text { rnos bier }\end{array}$ & $\begin{array}{l}\text { ajos que } \\
\text { entre nos }\end{array}$ & $\begin{array}{l}\text { acemos } \\
\text { tros }\end{array}$ & \\
\hline
\end{tabular}

Fuente: Elaboración propia con SPSS 25.

Desde la perspectiva del alumno de secundaria en Amozoc de Mota (Puebla), del 72\% de los alumnos que manifiestan tener muchos amigos en su escuela $(x=1.88$ y $\sigma=.831)$, el 99.3\% juegan mucho con ellos, en una escala de 1 (coeficiente de Pearson $\mathrm{r}=.835^{* *}$ y significativa al $\mathrm{p}=0.000$ ). Asimismo, los alumnos consideran que los profesores les enseñan a llevarse bien entre ellos. Con una media $\mathrm{x}=1.26$ y una desviación estándar $\sigma=.458$, con presencia de datos altamente dispersos y concentrados cercanos al promedio (coeficiente de Pearson $\mathrm{r}=.819^{* *}$ y significativa al $\mathrm{p}=0.000$ ). Y el profesor los motiva durante el trabajo diario $\mathrm{y}$ en las relaciones entre ellos, afirmado por el $99.3 \%$. Con una media $\mathrm{x}=1.35$ y una desviación estándar $\sigma=.493$, con presencia de datos altamente dispersos y concentrados cercanos al promedio (coeficiente de Pearson $\mathrm{r}=.819^{* *}$ y significativa al $\mathrm{p}=0.000$ ).

Finalmente, estos indicadores influyen en que los alumnos consideren que, solo el $49.3 \%$ que se lleva bien con sus compañeros de clase y el $50.7 \%$ se lleve regular. Con una media $\mathrm{x}=1.5 \mathrm{y}$ una desviación estándar $\sigma=.501$, con presencia de datos altamente dispersos y concentrados cercanos al promedio (coeficiente de Pearson $\mathrm{r}=.819 * *$ y significativa al $\mathrm{p}=$ 0.000). Y solo el 33.3\% se considera apreciado por sus compañeros (escala 1=Bien) y el 66\% no tan apreciados (escalla $2=$ regular), con una media $\mathrm{x}=1.67$ y una desviación estándar $\sigma=.484$, con presencia de datos altamente dispersos y concentrados cercanos al promedio (coeficiente de Pearson $\mathrm{r}=.740 * *$ y significativa al $\mathrm{p}=0.001$ ). Respecto a la Dimensión Autoconcepto del 
Alumno (ACA), y su relación con el Clima de Aula, con base en el análisis de correlaciones, se tiene que:

- Relación CA_A5 y CA_B1 : Del 36.6\% de alumnos que se auto-concibe como Valiente y del 61.3 como ni valiente ni miedoso $(2.1 \%=$ miedoso $)$, considera que está muy de acuerdo en que en general su relación con el profesor es satisfactoria (media $x=4.36$ y una desviación estándar $\sigma=.735$, con presencia de datos altamente dispersos y concentrados cercanos al promedio y relación lineal significativa positiva moderada (Johnson, 2003) con un coeficiente de Pearson $\mathrm{r}=.715^{* *}$ y significativa al $\mathrm{p}=0.000$ ).

- Relación CA_A6 y CA_B5 : De los alumnos que se auto-concibe como Peleonero= $7.3 \%$ y no Peleonero y no Pacífico=32.7\% (60\%=pacífico) considera que está muy de acuerdo en que en general su relación con sus compañeros es buena (media $\mathrm{x}=3.9$ y una desviación estándar $\sigma=.862$, con presencia de datos altamente dispersos y concentrados cercanos al promedio y relación lineal significativa positiva moderada (Johnson, 2003) con un coeficiente de Pearson $\mathrm{r}=.752 * *$ y significativa al $\mathrm{p}=0.000$ ).

- Relación CA_A7 y CA_B2: De los alumnos que se auto-concibe como Tristes=3.3\% y ni triste y ni alegre $=26.7 \%(70 \%=$ alegre $)$ considera que está muy de acuerdo en que en general la relación entre compañeros en el salón es buena (media $\mathrm{x}=3.88$ y una desviación estándar $\sigma=.862$, con presencia de datos altamente dispersos y concentrados cercanos al promedio y relación lineal significativa positiva moderada (Johnson, 2003) con un coeficiente de Pearson $\mathrm{r}=.919 * *$ y significativa al $\mathrm{p}=0.000$ ).

Respecto a la Dimensión Aceptación de las Normas de Conducta por parte del Alumno (ANCA), y su relación con el Clima de Aula, con base en el análisis de correlaciones, se tiene que:

- La Relación CA_A4 y las variables relacionadas con la aceptación de normas (CA_G1, CA_G2 y CA_G3): La correlación múltiple es negativa, es decir, el índice indica una dependencia total entre las variables es una relación inversa (cuando una de ellas aumenta, la otra disminuye en proporción constante): Cuando los alumnos/as agresivos acepten las normas de convivencia (CA_A4), entonces:

- Los alumnos cuando hagan algo que no deben (como pegar, insultar, faltar al respeto, etc) no estarán conscientes de lo que va a suceder o de las consecuencias (CA_G1). Es una Correlación lineal significativa negativa moderada (Johnson, 2003), donde r= $.661 * *$ y $\mathrm{p}=0.000$. 
- Los alumnos cuando en la escuela uno o varios de ellos peguen o insulten, percibirán que los profesores no intervinieran (CA_G2). Es una Correlación lineal significativa negativa moderada (Johnson, 2003), donde $\mathrm{r}=-.722 * * \mathrm{y} \mathrm{p}=0.000$.

- Los alumnos cuando en clase realicen actividades de integración, puede que reflexionen o no que el motivo es para aprender a llevarse bien entre ellos (CA_G3). Es una Correlación lineal significativa negativa moderada (Johnson, 2003), donde $\mathrm{r}=-.768 * *$ y $\mathrm{p}=0.000$.

\section{Resultados del análisis de ANOVA}

Se realizó la prueba ANOVA para la comparación de medias de tres intra-grupos clasificados como Clima de Aula Percibido por los Alumnos (CAPA), Autoconcepto del Alumno (ACA) y Aceptación de las Normas de Convivencia (ANCA). En la Tabla 6, se puede observar que el análisis se realizó bajo un diseño ex post facto retrospectivo (León \& Montero, 2003), que permitió analizar las medias de la Variable-Factor Clima de Aula (VFCA), procurando la mayor representatividad de la población de cada grupo (Altman \& Bland, 1996).

Tabla 6

Descriptivos variable-factor Clima de Aula (VFCA)

\begin{tabular}{|c|c|c|c|c|c|c|c|c|c|}
\hline & & \multirow[t]{2}{*}{$\mathbf{N}$} & \multirow[t]{2}{*}{ Media } & \multirow[t]{2}{*}{$\begin{array}{l}\text { Desviació } \\
\text { n típica }\end{array}$} & \multirow[t]{2}{*}{$\begin{array}{l}\text { Error } \\
\text { típico }\end{array}$} & \multicolumn{2}{|c|}{$\begin{array}{l}\text { Intervalo de confianza } \\
\text { para la media al } 95 \%\end{array}$} & \multirow[t]{2}{*}{ Mín. } & \multirow[t]{2}{*}{ Máx. } \\
\hline & & & & & & $\begin{array}{l}\text { Límite } \\
\text { inferior }\end{array}$ & $\begin{array}{l}\text { Límite } \\
\text { superior }\end{array}$ & & \\
\hline & 1.00 & 103 & 4.3592 & .48212 & .04750 & 4.2650 & 4.4534 & 4.00 & 5.00 \\
\hline CAPA & 2.00 & 46 & 2.8913 & .64043 & .09443 & 2.7011 & 3.0815 & 2.00 & 4.00 \\
\hline \multirow[t]{3}{*}{ CA_A7 } & 3.00 & 1 & 1.0000 & & & & & 1.00 & 1.00 \\
\hline & Total & 150 & 3.8867 & .89395 & .07299 & 3.7424 & 4.0309 & 1.00 & 5.00 \\
\hline & 1.00 & 103 & 4.4563 & .50052 & .04932 & 4.3585 & 4.5541 & 4.00 & 5.00 \\
\hline ANCA & 2.00 & 46 & 2.6087 & .77397 & .11412 & 2.3789 & 2.8385 & 1.00 & 4.00 \\
\hline \multirow[t]{3}{*}{ CA_A4 } & 3.00 & 1 & 1.0000 & & & & & 1.00 & 1.00 \\
\hline & Total & 150 & 3.8667 & 1.06605 & .08704 & 3.6947 & 4.0387 & 1.00 & 5.00 \\
\hline & 1.00 & 103 & 1.5534 & .49957 & .04922 & 1.4558 & 1.6510 & 1.00 & 2.00 \\
\hline $\mathrm{ACA}$ & 2.00 & 46 & 1.9565 & .29488 & .04348 & 1.8690 & 2.0441 & 1.00 & 3.00 \\
\hline \multirow[t]{2}{*}{ CA1_F1 } & 3.00 & 1 & 1.0000 & & & & & 1.00 & 1.00 \\
\hline & Total & 150 & 1.6733 & .48462 & .03957 & 1.5951 & 1.7515 & 1.00 & 3.00 \\
\hline
\end{tabular}

Fuente: Elaboración propia con SPSS 25.

Basados en la perspectiva de Blanco (2012) en sus estudios sobre el Clima Social Escolar, donde divide en dos subtemas para su mejor análisis: 1) en el mismo clima escolar y 2) en el clima de aula. Este último, tiene dos dimensiones desde la percepción de los profesores y desde la percepción de los alumnos, y se describe como las relaciones entre los alumnos y su profesor ya sea el tutor del grupo o del profesor de cualquier asignatura dentro del Aula (salón 
o laboratorio), afectan significativamente la autoconcepción de los alumnos y su nivel de aprendizaje (Link y Ratledge 1975; Alexander et al. 1987). Debido a que el Aula es un espacio de instrucción y de construcción del aprendizaje y de las relaciones sociales, ambos involucrados en el proceso educativo y sus resultados en el aprendizaje social, educativo, cognitivo y para la misma vida. Es así que, con base en la operacionalización de variables, se ha determinado las siguientes hipótesis:

- Las medias de los grupos $\mu 1$ (CAPA) $\mu 2$ (ACA) y $\mu 3$ (ANCA) son iguales y por tanto las diferencias encontradas pueden explicarse por el azar. Es decir, no hay diferencias entre el clima de aula percibido por los alumnos, ni en auto concepto del alumno y ni en la aceptación de las normas de conducta que afecten el clima de aula: Hipótesis nula (H0): $\mu 1=\mu 2=\mu 3$

- Las medias de los grupos $\mu 1$ (CAPA) $\mu 2$ (ACA) y $\mu 3$ (ANCA) no son iguales, o al menos uno de los grupos tiene una media distinta del resto de grupos. Es decir, si hay diferencias entre el clima de aula percibido, ni en auto concepto del alumno y ni en la aceptación de las normas de conducta que afecten el clima de aula: Hipótesis alternativa (H1): $\mu 1 \neq \mu 2 \neq \mu 3$.

Con base en los datos recabados y analizados con SPSS 25, para el análisis de ANOVA, se calcularon por grupo las Medias (M), la Desviación típica (DT), el Error estándar de la media (EEM) y el intervalo de confianza (IC). Como se puede observar en la Tabla 7, las medias comparadas son diferentes: Entre la variable-factor Clima de Aula (VFCA), entre los intragrupos CAPA, ACA y ANCA.

Tabla 7

Tabla ANOVA

\begin{tabular}{llrrrrr}
\hline & & $\begin{array}{c}\text { Suma de } \\
\text { cuadrados }\end{array}$ & gl & \multicolumn{1}{c}{$\begin{array}{c}\text { Media } \\
\text { cuadrática }\end{array}$} & F & Sig. \\
\hline CAPA & Inter-grupos & 76.908 & 2 & 38.454 & 134.062 & .000 \\
CA_A7 & Intra-grupos & 42.165 & 147 & .287 & & \\
& Total & 119.073 & 149 & & & \\
ANCA & Inter-grupos & 116.823 & 2 & 58.412 & 163.522 & .000 \\
CA_A4 & Intra-grupos & 52.510 & 147 & .357 & & \\
& Total & 169.333 & 149 & & & \\
ACA & Inter-grupos & 5.624 & 2 & 2.812 & 14.075 & .000 \\
CA1_F1 & Intra-grupos & 29.369 & 147 & .200 & & \\
& Total & 34.993 & 149 & & & \\
\hline
\end{tabular}

Fuente: Elaboración propia con SPSS 25. 
Asimismo, se reseña a continuación los siguientes datos descriptivos: Para la dimensión CAPA, la M es de 3.88, con una DT de .893 y EEM de .072. Es decir, el Clima de Aula Percibido es casi adecuado (aunque estando en la escala de 3, puede ser ni adecuado ni no adecuado).

- Para ACA, la M es de 1.67, con una DT de .484 y EEM de .039. Es decir, los alumnos se sienten aceptados por sus compañeros (aunque estando en la escala próxima a 2, puede que se sientan no aceptados).

- Para ANCA, la M es de 3.86, con una DT de 1.066 y EEM de .087. Es decir, los alumnos aceptan no del todo las normas de conducta (aunque estando en la escala 3, puede que sean indiferentes a la norma de la institución).

Como se puede observar el resultado de la ANOVA, calculado a través del programa SPSS 25. Se ha descompuesto la variabilidad total en dos filas: suma de cuadrados inter-grupos (entre los distintos grupos) y suma de cuadrados intra-grupos (dentro de cada grupo), divididas por sus correspondientes grados de libertad para obtener sus varianzas ANOVA (Stockburger, 1998 y Martínez, Sánchez-Villegas y Faulín, 2006): La suma de cuadrados inter-grupos con una media total de 76.908 para CAPA, de 116.82 para ANCA y de 5.624 para ACA. Una varianza inter-grupos de 38.454 para CAPA, de 58.412 para ANCA y de 2.812 para ACA. Una varianza intra-grupos de 0.287 para CAPA, de .357 para ANCA y de .200 para ACA. Y un cociente de varianzas $\mathrm{F}=134.062$ para $\mathrm{CAPA}$, de $\mathrm{F}=163.522$ para ANCA y de $\mathrm{F}=14.075$ para ACA. Da como resultado un valor de p asociada a F, con valor a 0.000 en las tres dimensiones de CAP.

Una vez que el valor de la distribución $\mathrm{F}$ es conocido y está tabulado en los tres grupos CAPA, ACA y ANCA, y las tres con una p asociada de 0.000. Se rechaza la hipótesis nula (Ho): $\mu 1=\mu 2=\mu 3$. Y se concluye que, si hay diferencias entre CAPA, ACA y ANCA. Debido a que existe diferencia en todas las medias de los grupos $\mu 1$ (CAPA) $\mu 2$ (ACA) у $\mu 3$ (ANCA). $\mathrm{O}$ al menos en un grupo cuya media es distinta a la media del resto de los grupos. Es decir, el Clima de Aula en las secundarias de la Zona Escolar 028 de Amozoc de Mota Puebla, si influye en el clima de aula percibido por el alumno, el autoconcepto que tienen y la aceptación de las normas de conducta en la convivencia escolar, ya que estas dimensiones son estadísticamente diferentes: Las medias de los grupos $\mu 1$ (CAPA) $\mu 2$ (ACA) y $\mu 3$ (ANCA) no son iguales, o al menos uno de los grupos tiene una media distinta del resto de grupos. Es decir, se acepta la 
hipótesis alternativa, si hay diferencias entre las medias de CAPA, ACA y ANCA $(\mathrm{H} 1: \mu 1 \neq$ $\mu 2 \neq \mu 3)$.

\section{Discusión}

De acuerdo con los resultados de este estudio descritos, se puede observar que el profesor de secundaria no se siente apreciado ni valorado por parte de los padres de familia, ni por sus autoridades en su centro de trabajo (director) y un poco menos por la supervisión escolar (en ese orden). Lo cual estrictamente no cumple con el objetivo del Plan Nacional de Convivencia Escolar, desde su perspectiva de Clima Social Escolar, debido a que no se es promueve ambientes de convivencia escolar armónica, pacífica e incluyente. El padre de familia es el núcleo y el pivote en la convencía escolar, porque la educación empieza en casa y en los padres recae la función de fomentar en sus hijos el respeto (a otros), la obediencia (adultos y autoridades), la regulación de las emociones (la ira, el enojo y las emociones de frustración) en la convivencia con adultos y sus compañeros, tanto en la escuela como en sus relaciones fuera de ella (en casa, en los trabajos, en la sociedad en general).

Asimismo, el fortalecimiento de los valores familiares se debe dar a partir de esta nueva tendencia en la composición familiar. Tanto en México como en otros países, existen 11 tipos de familias (desde el tipo clásica hasta las formadas por padres/madres del mismo sexo con hijos, con un solo padre o madre con hijos hasta familias con perros que tratan y viven como si fueran sus hijos), por ende, el aprecio por la diversidad y el fortalecimiento de los valores familiares (Trabajo integral con la familia) es fundamento del clima social escolar en sus dos perspectivas: el clima escolar y el clima en el aula (Tuvilla, 2004; Pineda-Alfonso y GarcíaPérez, 2016; Celkan et al., 2015; Gómez, 2017). Porque los hijos, los alumnos replican lo vivido en casa (aprendizajes, formas de pensar y formas de vivir) y lo fortalecen o modifican a partir de lo aprendido en clase, en la escuela a través de la enseñanza y el ejemplo del profesor.

En este último aspecto, en los resultados analizados se puede observar que el profesor de secundaria no siente el apoyo ni se siente valorado por parte de sus compañeros de trabajo (colegas o pares y por parte de los directivos). El profesor es un agente de andamiaje en el clima escolar, quien debe promover el desarrollo de los alumnos desde una perspectiva personal, social y académica. Si bien él también es parte de las estratégicas del PNCE, en el establecimiento de vínculos positivos entre los alumnos y los profesores. Es también, el promotor de un clima afectuoso y de seguridad, con normas y límites claros, con el ejemplo que da en sus relaciones con sus homólogos en la escuela. Los alumnos son testigos del desempeño 
emocional y de convivencia del docente para con sus autoridades, sus pares y los padres de familia.

Las limitaciones del estudio se presentan desde la perspectiva del clima escolar, cuando se es posible un análisis de la satisfacción escolar en los docentes, alumnos y padres de familia. En este último rubro, es preciso considerar la revisión del contenido de los factores desde la opinión de los padres de familia, ya que la educación y la formación que se genera desde casa, desde la familia. Y es donde recae las implicancias para los estudios futuros del tema de investigación, Con la formación de una adecuada convivencia para brindar las bases necesarias para presentar en los establecimientos educativos los resultados y someterlos a contraste empírico. Con ayuda del análisis de ecuaciones estructurales que se revela como un instrumento adecuado para el tipo de ajustes del futuro de esta investigación.

\section{Conclusiones}

Con base en los resultados analizados y en las interpretaciones de los comportamientos de los alumnos se secundaria de la zona escolar 038 en Amozoc de Mota Puebla, podemos concluir que el rol del docente dentro del aula es de primordial importancia en el clima de aula que perciban los alumnos. Los alumnos se sienten bien entre ellos, aceptados y respetados con base en la postura conciliadora, interventora y de autoridad del profesor. Además de presentar una imagen de preocupación hacia el alumno, que lo motiva no solo a asistir a la escuela, sino que también le refuerza la autoestima, la valentía y el respeto por sus compañeros. Con la presente investigación, nos podemos dar cuenta que, si el profesor es un mal ejemplo de convivencia con sus pares y autoridades, el alumno tomará el mismo rol con sus compañeros. Si bien, parte de los valores de conducta vienen de casa, el alumno los reforzará o evitará con base a los valores que presente, represente y actúe el profesor dentro, fuera y alrededor el aula. Aspecto importante que preocupa y ocupa en estrategias al Programa Nacional de Convivencia Escolar (PNCE). Pero también debe de hacer reflexionar al mismo profesor, sobre su labor social y, académica que tiene con el alumno. No solo es impartir clases y controlar un grupo de jóvenes, es reflexionar sobre el rol de formador, instructor y agente de cambio en la vida diaria del adolescente, que adolece de una identidad, madurez y control de sus emociones.

Finalmente, el Clima Escolar como el clima de aula, se construye por la calidad de las relaciones entre los agentes educativos, como son las autoridades educativas (secretarios de gobierno, directores de sección, jefes y supervisores), las autoridades escolares en la escuela misma (directores), el personal administrativo (secretarias y asistentes), los padres de familia y 
los alumnos. Entonces, para construir un clima social-escolar afectivo, se requiere de convenciones sociales y hábitos de cortesía, la promoción de valores éticos y el cuidado del otro (en la convivencia) y las relaciones familiares (en la administración y sostenimiento de la escuela. Con la implementación de estrategias de Marketing Educativo se puede ayudar a mejorar la convivencia y promover las relaciones basadas en el respeto y la aceptación. Fortalecido en con los planes y programas educativos y las estrategias de convivencia escolar, lo que desarrollará en los alumnos, docentes, directivos y padres de familia actitudes asertivas, críticas, con valores y conciencia analítica.

\section{Referencias}

Aguilera, M.A., (2015). La violencia/convivencia escolar y la calidad educativa, Proceso de armonización de la ley general de educación con la ley general de los derechos de niñas, niños y adolescentes. INEE, México. [En línea]. Disponible en: https://www.senado.gob.mx/comisiones/educacion/Escuela_Libre_Violencia/docs/con vivencia.pdf.

Alexander, K.; Entwisle, D. y Thompson, M., (1987). School performance: status relations, and the structure of sentiment: bringing the teacher back in. American sociological review. Vol. 52, núm. 5: 665-682. DOI: https://doi.org/10.2307/2095602.

Altman, D. G., \& Bland, J. M. (1996). Statistics Notes: Comparing several groups using analysis of variance. Bmj, 312(7044), 1472-1473. DOI: https://doi.org/10.1136/bmj.312.7044.1472.

Benbenishty, R., Astor, R. A., Roziner, I., \& Wrabel, S. L. (2016). Testing the causal links between school climate, school violence, and school academic performance: A crosslagged panel autoregressive model. Educational Researcher, 45(3), 197-206. DOI: https://doi.org/10.3102/0013189X16644603.

Blanco Bosco, E. (2009). La desigualdad de resultados educativos: aportes a la teoría desde la investigación sobre eficacia escolar. Revista mexicana de investigación educativa, 14(43), 1019-1049. [En línea]. Disponible en: http://www.scielo.org.mx/scielo.php?pid=S1405$66662009000400003 \&$ script=sci_arttext.

Blanco. E. B. (2012). La relación entre el clima escolar y los aprendizajes en México. In Ponencia presentada en el X Congreso Nacional de Investigación Educativa. Mesa (Vol. 10). [En línea]. Disponible en: http://formaciondocente.com.mx/Bibliotecadigital/01_AfectividadConvivencia/La\%20 Relacion\%20Entre\%20el\%20Clima\%20Escolar\%20y\%201os\%20Aprendizajes\%20en \%20Mexico.pdf

Braghirolli, L., Ribeiro, J. L., Weise, A. D. y Pizzolato, M. (2016). Benefits of educational games as an introductory activity in industrial engineering education. Computers in Human Behavior, 58, 315-324. DOI: https://10.1016/j.chb.2015.12.063. 
Brennan, R. y Vos, L., (2013). Effects of participation in a simulation game on marketing students' numeracy and financial skills. Journal of Marketing Education, 35(3), 259270. DOI: https://10.1177/0273475313482928.

Caruana, A., \& Calleya, P., (1998). The effect of internal marketing on organizational commitment among retail bank managers. International Journal of Bank Marketing, 16, 108-116. DOI: https://doi.org/10.1108/02652329810213510.

Celkan, G., L. Green y K. Hussain, (2015). Student Perceptions of Teacher Respect Toward College Students, Procedia-Social and Behavioral Sciences, 191, 2174-2178. DOI: https://doi.org/10.1016/j.sbspro.2015.04.435.

Collie, R. J., Shapka, J. D., \& Perry, N. E. (2011). Predicting teacher commitment: The impact of school climate and social-emotional learning. Psychology in the Schools, 48(10), 1034-1048. DOI: https://doi.org/10.1002/pits.20611.

Dávila Balcarce, G., \& Velásquez Contreras, Á. (2007). Evaluación de la aplicación de juegos colaborativos:" Devorón" y" Temporal". Revista electrónica de investigación educativa, 9(2), 1-20.). [En línea]. en: http://www.scielo.org.mx/scielo.php?script=sci_arttext\&pid=S1607$\underline{40412007000200005 .}$.

Edmonds, R. (1979). Effective schools for the urban poor. Educational leadership, 37(1), 1524. [En línea]. Disponible en: http://www.brjonesphd.com/uploads/1/6/9/4/16946150/effective_schools__ron_edmunds.pdf.

Espelage, D. L., Low, S. K., \& Jimerson, S. R. (2014). Understanding school climate, aggression, peer victimization, and bully perpetration: Contemporary science, practice, and policy. School Psychology Quarterly, 29(3), 233-237. DOI: https://doi.org/10.1037/spq0000090.

Ewing, M. T., \& Caruana, A., (1999). An internal marketing approach to public sector management: The marketing and human resource interface. International Journal of Public Sector Management, 12, 17-29. DOI: https://doi.org/10.1186/1472-6920-14-50.

Forrester, J. W. (1959). Advertising: a problem in industrial dynamics. Harvard Business Review, 37, 100-110. [En línea]. Disponible en: https://ci.nii.ac.jp/naid/10026017444/.

Gómez, E. L. (2017). Educación en valores. Revista Raites, 3(6). [En línea]. Disponible en: http://www.itcelaya.edu.mx/ojs/index.php/raites/article/view/720.

Good, C. P., McIntosh, K., \& Gietz, C. (2011). Integrating bullying prevention into schoolwide positive behavior support. Teaching Exceptional Children, 44(1), 48-56. DOI: https://doi.org/10.1177/004005991104400106.

Greene, M. B. (2006). Bullying in schools: A plea for measure of human rights. Journal of Social Issues, 62(1), 63-79. DOI: https://doi.org/10.1111/j.1540-4560.2006.00439.x.

INEE - Instituto Nacional para la Evaluación Educativa, (2018). Panorama Educativo de México 2017. Indicadores del Sistema Educativo Nacional. Educación Básica y Media 
Superior. México: Autor. [En línea]. Disponible en: https://www.inee.edu.mx/wpcontent/uploads/2019/08/P1B117.pdf.

Johlke, M. C., \& Duhan, D. F. (2000). Supervisor communication practices and service employee job outcomes. Journal of Service Research, 3(2), 154-165. DOI: https://doi.org/10.1177/109467050032004.

Johnson, R., (2003). Estadística Elemental, Ed. Math Learning, Ed. Tercera: México DF.

Johnson, S. L. (2009). Improving the school environment to reduce school violence: A review of the literature. Journal of school health, 79(10), 451-465. DOI: https://doi.org/10.1111/j.1746-1561.2009.00435.x.

Kerlinger, F. y Lee. (2202). Investigación del comportamiento. México: Mc Graw-Hill.

Konishi, C., Miyazaki, Y., Hymel, S., \& Waterhouse, T. (2017). Investigating associations between school climate and bullying in secondary schools: Multilevel contextual effects modeling. School Psychology International, 38(3), 240-263. DOI: https://doi.org/10.1177/0143034316688730.

Kotler, P. (2000). Marketing Management. New Jersey: Prentice Hall.

Landeros, L., \& Chávez, C. (2015). Convivencia y disciplina en la escuela: análisis de reglamentos escolares de México. Instituto Nacional para la Evaluación de la Educación. [En línea]. Disponible en: https://www.inee.edu.mx/wpcontent/uploads/2019/01/Sesion_5_Concepcion_Chavez.pdf.

Larios-Gómez, E. (2015). Mercadotecnia Educativa: Un Modelo Latinoamericano. Caderno Profissional de Marketing-UNIMEP, 3(1), 14-33. [En línea]. Disponible en: https://www.cadernomarketingunimep.com.br/ojs/index.php/cadprofmkt/article/view/3 1.

Larios-Gómez, E.; Larios, G.J.; \& Peña, L.B., (2018). Gestión Educativa: Un estudio de caso del clima escolar y del aula en la escuela secundaria en Puebla. En Larios-Gómez, E.;Vázquez, F. E., \& Giuliani, A.C. (Coords.). Administración Ciencia de Gestión Aplicada (pp. 193-207), México: Pearson.

Lázaro-Visa, S., \& Fernández-Fuertes, A. A. (2018). Clima escolar, conflicto y gestión de la convivencia en los centros educativos de Cantabria. Santander. Gobierno de Cantabria, Consejería de Educación, Cultura y Deporte, DL: SA-292-2018 - ISBN: 978-84-0902189-5: 29-56. Cantabria España: Universidad de Cantabria Ed. Disponible en: https://1library.co/document/ozldwm6y-clima-escolar-conflicto-gestion-convivenciacentros-educativos.html.

Lee, V. E., \& Bryk, A. S. (1989). A multilevel model of the social distribution of high school achievement. Sociology of education, 172-192. DOI: https://doi.org/10.2307/2112866.

Lee, V. E., Smith, J. B., \& Croninger, R. G. (1997). How high school organization influences the equitable distribution of learning in mathematics and science. Sociology of education, 128-150. DOI: https://doi.org/10.2307/2673160. [En línea]. Disponible en: https://www.jstor.org/stable/2673160. 
León, O. G. y Montero, I. (2003). Métodos de investigación en Psicología y Educación ( $3^{\mathrm{a}}$ ed.). Madrid: McGraw-Hill.

Lings, I., \& Greenley, G. E., (2005). Measuring internal market orientation. Journal of Service Research, 7, 290-305. DOI: https://doi.org/10.1177/1094670504271154.

Link, C. R., \& Ratledge, E. C. (1979). Student perceptions, IQ and achievement. Journal of Human Resources, 98-111. DOI: https://doi.org/10.2307/145540.

Loukas, A., \& Murphy, J. L. (2007). Middle school student perceptions of school climate: Examining protective functions on subsequent adjustment problems. Journal of school psychology, 45(3), 293-309. DOI: https://doi.org/10.1016/j.jsp.2006.10.001.

Low, S., \& Van Ryzin, M. (2014). The moderating effects of school climate on bullying prevention efforts. School Psychology Quarterly, 29(3), 306. DOI: https://doi.org/10.1037/spq0000073.

Malhotra, N. K., Kim, S. S., \& Patil, A. (2006). Common method variance in IS research: A comparison of alternative approaches and a reanalysis of past research. Management science, 52(12), 1865-1883. DOI: https://doi.org/10.1287/mnsc.1060.0597.

March, J. (1962). The business firm a political coalition. The Journal of Politics, 24(4), 662678. DOI: https://10.1017/S0022381600016169.

Mateos-Aparicio, G. y Martín, M. (2002). El análisis de la varianza en la investigación comercial. Madrid: Prentice Hall.

McRaith, J. F., Goeldner, C. R., \& Ferber, R. (1962). A survey of marketing games. Journal of Marketing, 26(3), 69-72. DOI: https://doi.org/10.1177/002224296202600314.

Montes, D. A., \& Suárez, C. I. (2016). La formación docente universitaria: claves formativas de universidades españolas. Revista electrónica de investigación educativa, 18(3), 5164. [En línea]. Disponible en: http://www.scielo.org.mx/scielo.php?pid=S1607$\underline{40412016000300004 \& \text { script=sci_arttext. }}$.

Montoya Suárez O. (2007). Aplicación del análisis factorial a la investigación de mercados. Caso de estudio. Scientia Et Technica, 1(35). https://doi.org/10.22517/23447214.5443.

Nunnally, J. C. (1978). Psychometric theory (2nd ed.). New York: McGraw-Hill.

Pertegal, M. A., \& Hernando, A. (2015). El contexto escolar como promotor del desarrollo positivo adolescente. Madrid: Síntesis.

Pineda-Alfonso, J. A., \& García-Pérez, F. F. (2016). Conflicto y convivencia: Profesores y alumnos en el proceso de enseñanza en un aula de secundaria. Revista mexicana de investigación educativa, 21(71), 1073-1091. [En línea]. Disponible en: http://www.scielo.org.mx/scielo.php?script=sci_arttext\&pid=S1405$\underline{66662016000401073 .}$

PNCE -Programa Nacional de Convivencia Escolar (2017). Gobierno de la República. [En línea]. Disponible en: https://www.gob.mx/escuelalibredeacoso/articulos/programanacional-de-convivencia-escolar-120992. 
PND -Plan Nacional de Desarrollo 2013-2018 (2013). Gobierno de la República. [En línea]. Disponible en: http://www.dof.gob.mx/nota_detalle_popup.php?codigo=5299465.

Qian, M. y Clark, K. R. (2016). Game-based learning and 21st century skills: a review of recent research. Computers in Human Behavior, 63, 50-58. DOI: https://doi.org/10.1016/j.chb.2016.05.023.

INEE (2019). Panorama Educativo de México 2018. Indicadores del Sistema Educativo Nacional. Educación básica y media superior. México: autor.

Schultz, D. E. (2002). Study internal marketing for better impact. Marketing News, 36(21), 810.

SEB -Subsecretaría de educación básica (2016). Programa nacional de convivencia escolar. Dirección general de desarrollo de la gestión educativa. Disponible en: https://www.gob.mx/cms/uploads/attachment/file/195743/DB_PNCE_260217.pdf.

Shycon, H. N., \& Maffei, R. B. (1960). Simulation-tool for better distribution. Shycon, H. N., \& Maffei, R. B. (1960). Simulation-tool for better distribution. Harvard Business Review, 38(6), 6575. (6), 65-75.

Stephenson, P., \& Smith, D. (2008). Por qué algunas escuelas no tienen acosadores. In Intimidación: una guía práctica para combatir el miedo en las escuelas (pp. 56-78). Fondo de Cultura Económica.

Stockburger, D.W. ANOVA. (1998). Why Multiple Comparisons Using t-tests is NOT the Analysis of Choice. [En línea]. Disponible en: http://davidmlane.com/hyperstat/intro_ANOVA.html.

Titus, P. A., \& Petroshius, S. M. (1993). Bringing consumer behavior to the workbench: An experiential approach. Journal of Marketing Education, 15(1), 20-30. DOI: https://doi.org/10.1177/027347539301500104.

Tuvilla Rayo, J. (2004). Convivencia escolar y resolución pacífica de conflictos. Junta de Andalucía, Consejería de educación y Ciencia. Dirección general de educación orientativa y solidaridad. Plan Andaluz de educación para la cultura de paz y No violencia. Artes Gráficas Gandolfo. [En línea]. Disponible en: https://diversidad.murciaeduca.es/orientamur/gestion/documentos/5._convivenciaandalucia.pdf.

Santos, J., \& Gonçalves, G. M. R. (2018). Organizational culture, internal marketing, and perceived organizational support in Portuguese higher education institutions. Journal of Work and Organizational Psychology-Revista De Psicologia Del Trabajo Y De Las Organizaciones, 34(1), 38-45. DOI: https:// 10.5093/jwop2018a5.

Voight, A., \& Nation, M. (2016). Practices for improving secondary school climate: A systematic review of the research literature. American Journal of Community Psychology, 58(1-2), 174-191. DOI: https://doi.org/10.1002/ajcp.12074. 
Vos, L. (2015). Simulation games in business and marketing education: How educators assess student learning from simulations. The International Journal of Management Education, 13(1), 57-74. DOI: https://10.1016/j.ijme.2015.01.001. 